\title{
Histological Modifications of Postmenopausal Vaginal Mucosa after Regenerative Solid State Laser Treatment: A Multicenter Study
}

\author{
D Dodero ${ }^{1}$, F Frascani ${ }^{2}$, M Angelucci ${ }^{3}$, G Bernabei ${ }^{4}$, E Merlo ${ }^{1}$, F Locatelli ${ }^{4}$, D Recalcati and F Murina $^{5, *}$ \\ ${ }^{1}$ Villa Maris Clinic in Genova, Italy \\ ${ }^{2}$ Casa di Cura Santa Famiglia in Rome, Italy \\ ${ }^{3}$ Blu Mar Clinic in Milan, Italy \\ ${ }^{4}$ Studio Locatelli in Modena and Lower Genital Tract Disease Unit, Italy \\ ${ }^{5}$ Buzzi Hospital, University of Milan, Italy
}

*Corresponding author: Filippo Murina, Lower Tract Disease Unit, Buzzi Hospital, University of Milan, via Castelvetro 32, Milano, Italy

\section{Abstract}

Background: Vulvovaginal atrophy (VVA) is a condition afflicting more than $50 \%$ of women and nowadays has changed his name in Genitourinary syndrome of menopause (GSM) due to the multiple aspects involved having an adverse impact on quality of life. Many treatment are now available and laser represent a new powerful option. The aim of this work is assess the histological effect of a new type of non-ablative laser, Solid State Vaginal Laser (SSVL), on vaginal tissue in women treated for GSM.

Method: Eighty participants with GSM symptoms were treated with a total of 4 treatments in a two months timeframe (every 15-20 day) of a non-ablative SSVL (LASEmaR $1500^{\mathrm{TM}}$ - EUFOTON). Vaginal biopic samples were collected before the start of the first treatment and after 4 weeks from the last one and an analysis of histological characteristics was conducted. A cumulative intensity of GSM symptoms using a 10-cm VAS (dryness and/or burning and/or dyspareunia), the vaginal health index (VHI), the Female Sexual Function Index (FSFI) and Urinary Incontinence Short Form (ICIQ-UI SF) were also evaluated.

Results: Improvement in vaginal histological parameters for atrophy was demonstrated following the SSVL treatment. Amelioration was observed in order of thickening of the derma surface, phenomenon of glycogenic acanthosis, increased number and highness of papillae in chorion and in term of reduction in the inflammatory tissue infiltrate. Was also observed on VHIS, VVA symptoms and sexual female function.
\end{abstract}

Conclusion: The evaluation of histological results indicates a favorable effect of SSVL on vaginal atrophy in GSM in terms of epithelium restauration as well as on inflammatory infiltration of the tissue.

\section{Keywords}

Atrophic vaginitis, Genitourinary syndrome of menopause, Urinary incontinence, Fractional laser

\begin{abstract}
Abbreviations
GSM: Genitourinary Syndrome of Menopause; VVA: Vulvovaginal Atrophy; SSVL: Solid State Vaginal Laser; VHI: Vaginal Health Index; FSFI: Female Sexual Function Index; ICIQ-UI SF: Urinary Incontinence Short Form; VAS: Visual Analytic Scale
\end{abstract}

\section{Introduction}

Vulvovaginal atrophy (VVA) is a condition afflicting more than $50 \%$ of women and nowadays has changed his name in Genitourinary Syndrome of Menopause (GSM) due to the multiple aspects involved having an adverse impact on quality of life. This is a condition occurring at menopause due to low levels of estrogen [1] and the definition GSM defines better than VVA all the possible symptoms and clinical signs from the lower genital (VVA) and urinary tract system (LUTS) [1]. These

Citation: Dodero D, Frascani F, Angelucci M, Bernabei G, Merlo E, et al. (2019) Histological Modifications of Postmenopausal Vaginal Mucosa after Regenerative Solid State Laser Treatment: A Multicenter Study. Int J Womens Health Wellness 5:099. doi.org/10.23937/2474-1353/1510099

Accepted: July 15, 2019: Published: July 17, 2019

Copyright: (c) 2019 Dodero D, et al. This is an open-access article distributed under the terms of the Creative Commons Attribution License, which permits unrestricted use, distribution, and reproduction in any medium, provided the original author and source are credited. 
women may present with one or more symptoms as dyspareunia, dryness, itching/burning, sexual dysfunction, dysuria, urinary frequency, and urgency and all of these symptoms present atrophy as primo movens [2]. In order of atrophy's degree can be present vaginal dryness in $75 \%$ postmenopausal women, dyspareunia (38\%) and vaginal itching, discharge, and pain (15\%) [3]. Topical hormonal treatment is still considered the gold standard therapy to restore from atrophy and consequently for postmenopausal related vaginal symptoms, based on restoration of epithelial integrity and vaginal flora [4]. This treatment needs unfortunately multiple self-applications during time and sometime is associated with increased vaginal discharge, all of these ending in poor patient's compliance. The prescription of topical estrogens has also to be avoided in women with history of breast cancer, estrogen-sensitive tumors, and thromboembolism, emphasizing the necessity for treatment alternatives [5]. When dryness is the only symptom and option can be represented by lubricants and moisturizers, but usually they are not enough to reduce vulvar pain and sexual discomfort $[6,7]$.

A new option can now be represented by lasers due to their capacity in promoting revitalization of the tissue that ends in a 'rejuvenation' effect of vaginal and vulvar tissue having as a target the collagen and fibroblastic activity. The amelioration in the atrophy condition can be the first step in improving the related symptoms, vaginal laxity, stress urinary incontinence, sexual dysfunction. The quality of data supporting the use of vaginal lasers in GSM is becoming stronger and stronger as new studies are conducted [8]. The aim of this paper is now to demonstrate the histological change in vaginal tissue that lead to regeneration and rejuvenation effect, due to the laser application and, to do this, we used a new type of non-ablative laser, Solid State Vaginal Laser. In addition a concomitant reduction in the inflammatory infiltrate was demonstrated. This is a data subanalysis of a more complex study regarding GSM's symptoms in their wholeness where were also evaluated the vaginal health index (VHI), the Female Sexual Function Index (FSFI) and Urinary Incontinence Short Form (ICIQ-UI SF).

\section{Methods}

\section{Study design}

This prospective observational multicenter study included postmenopausal women (absence of menstruation for at least 12 months) presenting with symptoms of GSM (vaginal dryness, irritation, soreness, dyspareunia and urinary incontinence), and not responding/being unsatisfied with previous local estrogen therapies.

Study exclusion were use of any hormone replacement therapies (either systemic or local) within the 6 months prior to inclusion in the study, use of vaginal moisturizers, lubricants or any other local preparation within the 30 days prior to inclusion in the study, acute or recurrent urinary tract and genital infections, serious or chronic illness that could interfere with the fulfillment of the study and psychiatric disorders precluding informed consent. The study protocol was approved by the Local Ethics Committee, and informed written consent was obtained from study subjects.

\section{Study protocol}

All patients were treated with the non-ablative SSVL (LASEmaR $1500^{\mathrm{TM}}$ - EUFOTON) with a wavelength of $1470 \mathrm{~nm}$ using a fluence (laser energy emitted per unit area) of $10-15 \mathrm{~J} / \mathrm{cm}^{2}$. The handpiece of the SSVL (LADYLIFT) used to perform the treatment is a vaginal internal probe developed for gentle introduction with a radial light emission in continuous mode working at $360^{\circ}$ on the vaginal channel. Its spot size it's larger than the spot size of the other vaginal laser $\left(\mathrm{CO}_{2}, \mathrm{Er}\right.$ : Yag spot size 150-300 micron) and this characteristic enables greater depth of penetration improving the absorption of energy at the target. Particularly this laser uses a laser wavelength with a proper mix between water absorption and tissue penetration without creating ablation. All eligible patients were submitted to vaginal biopsy before and after SSVL treatment to evaluate microscopic and ultrastructural modifications of vaginal mucosa.

According to our protocol, a treatment cycle included a laser application every 15-20 day for a total of 4 treatments in about two months. Time points of the study were at baseline (T0), at week 2 (T1), at week 4 (T2), at week 6 (T3), at week 8 (T4), and a follow-up after 4 weeks from the last laser application (T5). The procedure was performed in the outpatient clinic and did not require any specific preparation (e.g. analgesia/anesthesia). After each treatment the patients were advised not to have sexual activity and not to wear tight clothing and/or play sports that would rub the treated area for 1 week. We performed a bioptic vaginal sample before the start of the first treatment (TO) and after 4 weeks from the last treatment (T5).

\section{Data assessments}

Histological findings for atrophy where analyzed using vaginal bioptic samples for each patient collected at baseline (TO) and after 4 weeks from the last treatment (T5). Thickness of the epithelium, presence or increase in papillae, fibrosis of chorion and inflammatory infiltrated were analyzed. Also the correlation with the symptoms was investigated: Participants reported a cumulative intensity of GSM symptoms using a 10-cm VAS (dryness and/or burning and/or dyspareunia). The scale's left extremity indicates the complete absence of symptoms (0) and the right extremity indicates the worst possible symptom, and women rated the symptoms from 0 to 10 . The vaginal health index (VHI), a quantitative assessment of vaginal health, was performed by the investigator to assess chang- 
Table 1: Patients demographics and baselines characteristics.

\begin{tabular}{|l|l|}
\hline Age $(\mathrm{y})$ & $54.2( \pm 5.2)$ \\
\hline BMI $\left(\mathrm{kg} / \mathrm{m}^{2}\right)$ & $25.3( \pm 1.2)$ \\
\hline Smoke yes - no & $\begin{array}{l}38(47.5 \%)-42 \\
(52.5 \%)\end{array}$ \\
\hline Patients with previous breast cancer & $4(5.0 \%)$ \\
\hline Parity & $2(1-3)$ \\
\hline Sexual active women & $72(90.0 \%)$ \\
\hline Previous systemic HRT & $33(41.0 \%)$ \\
\hline Previous local HRT & $50(63.0 \%)$ \\
\hline Duration of previous HRT (months) & $33(6-60)$ \\
\hline & Total patients n 80 \\
\hline
\end{tabular}

es in vaginal elasticity, fluid volume, vaginal $\mathrm{pH}$ level, and epithelial integrity and moisture after treatment and at follow-ups after the final treatment $(6,12$, and 24 weeks) compared to baseline. Each parameter was graded from 1 to 5 , being atrophic a total score $\leq 15$ [9]. The Female Sexual Function Index (FSFI) questionnaire was also administered. The FSFI is a 19-item, multidimensional self-report instrument for assessing the key dimensions of sexual function in women [10]. The secondary outcome was to analyze the efficacy of SSVL in patients affected by urge/stress incontinence using International Consultation on Incontinence Questionnaire - Urinary Incontinence Short Form (ICIQ-UI SF). The ICIQ-UI SF provides a brief and robust measure to assess the impact of symptoms of incontinence on quality of life and outcome of treatment [11].

It is a four diagnostic items questionnaire about frequency of urinary incontinence, amount of leakage, overall impact of urinary incontinence and self-diagnostic items that is used to screen for incontinence, to obtain a brief yet comprehensive summary of the level, impact and perceived cause of symptoms of incontinence. The scoring system consists in 0-21 overall score with greater values indicating increased severity and self-diagnostic item unscored. The test is considered normal when the ICIQ-UI SF score is $<11$.

Differences between values at baseline and followup were analyzed with Wilcoxon signed-rank test for paired data. Statistical significance was set at $P<0.05$. First, confirm that you have the correct template for your paper size. This template has been tailored for output on the custom paper size $(21 \mathrm{~cm} \times 28.5 \mathrm{~cm})$.

\section{Results}

Eighty participants (mean age $57.2 \pm 5.4$ years) were enrolled and their baseline characteristics are showed in Table 1. None of the participants presented any serious side effects due to the treatment. Only a temporary mild irritation of the introitus was reported, starting immediately after the laser treatment, and lasting 2 hours long, not requiring any medications.

The analysis of the vaginal tissue samples collected

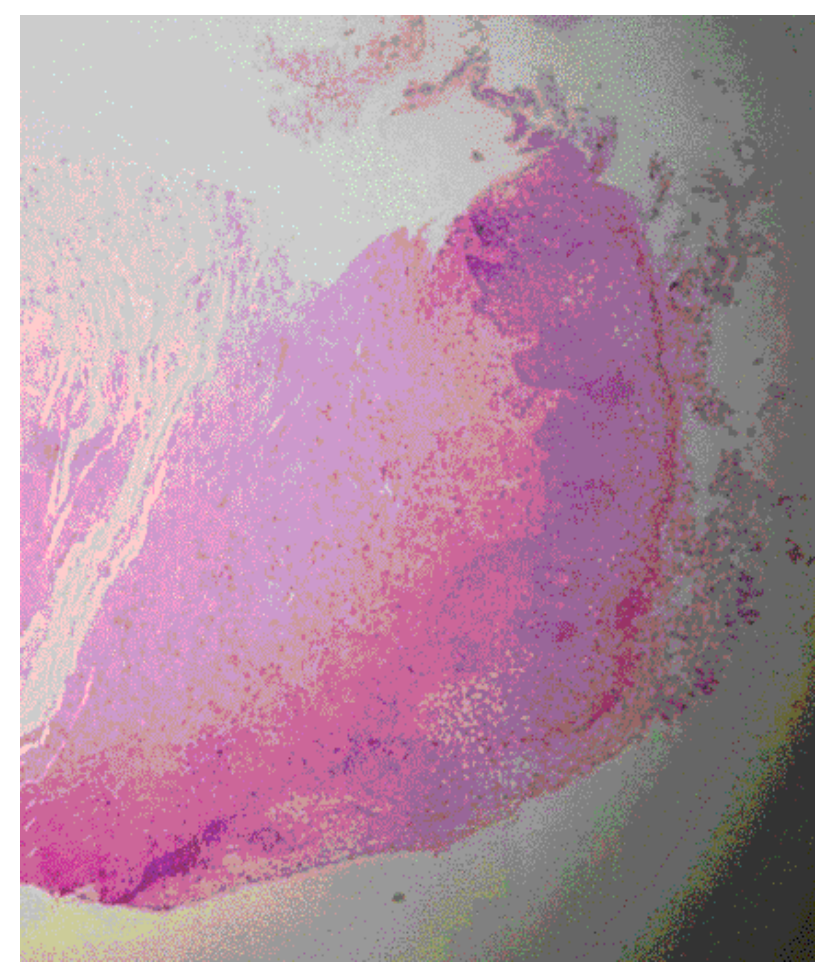

Figure 1: Vaginal sample at T0: Vaginal Histology at baseline.

at T0 showed signs of atrophic vulvo-vaginitis at different stages, but all samples presented a remarkable thinning of vulvo-vaginal epithelium, a reduction or disappearance of papillae and a marked fibrosis of chorion. In addition, a significant reduction in collagen activity was observed accompanied by an increase of inflammatory infiltrate, predominantly lymphocytic, and in few cases the typical lichenoid "band" arrangement could be seen Figure 1.

After four laser treatments (T4), study protocol complete, new biopsies were taken and the new vulvo-vaginal samples show almost a restauration to "normal" epithelium or, more correctly, to a pre-menopausal histological condition. As show in Figure 2 the coating of the epithelium is evident, the thickening of the derma surface con be observed together with a phenomenon as the glycogenic acanthosis causing the "glycogenization", a benefic process inducted by laser treatment. In addition, in the chorion we assisted to a gain in number and highness of papillae hand in hand with reduction of fibrosis. At last an increasing in fibroblastic activity was demonstrated generating a restauration in collagenic structure and causing angioblastic remodulation as can be seen with the appearance of what is called "angioblastic granulation tissue-like" Figure 2 .

The other key point of this study is the histological demonstration of inflammatory reduction: Comparing the tissues collected at T4 with T0 there is a decreasing in the lymphocytic and, in general in all inflammatory infiltrate of derma and chorion, almost none in T4, ending in a restitution ad-integrum also from an inflammation point of view. This is probably one of the main point act- 
ing in the GSM symptoms reduction.

In fact the severity and the presence of GSMsymptoms decreased significantly while the treatment went by and also the sexual function (as assessed by the FSFI) improved significantly (Table 2). At T4 70\% of patients had no longer any symptoms; $15 \%$ declared clear improvement; $10 \%$ partial improvement; $5 \%$ little benefit and none a worsening condition. No one reported side effects lasting or appeared after laser treatment.

These results were maintained after the end of treatment and were unvaried at four weeks distance (T5).

Also the self-perception of atrophy, measured with

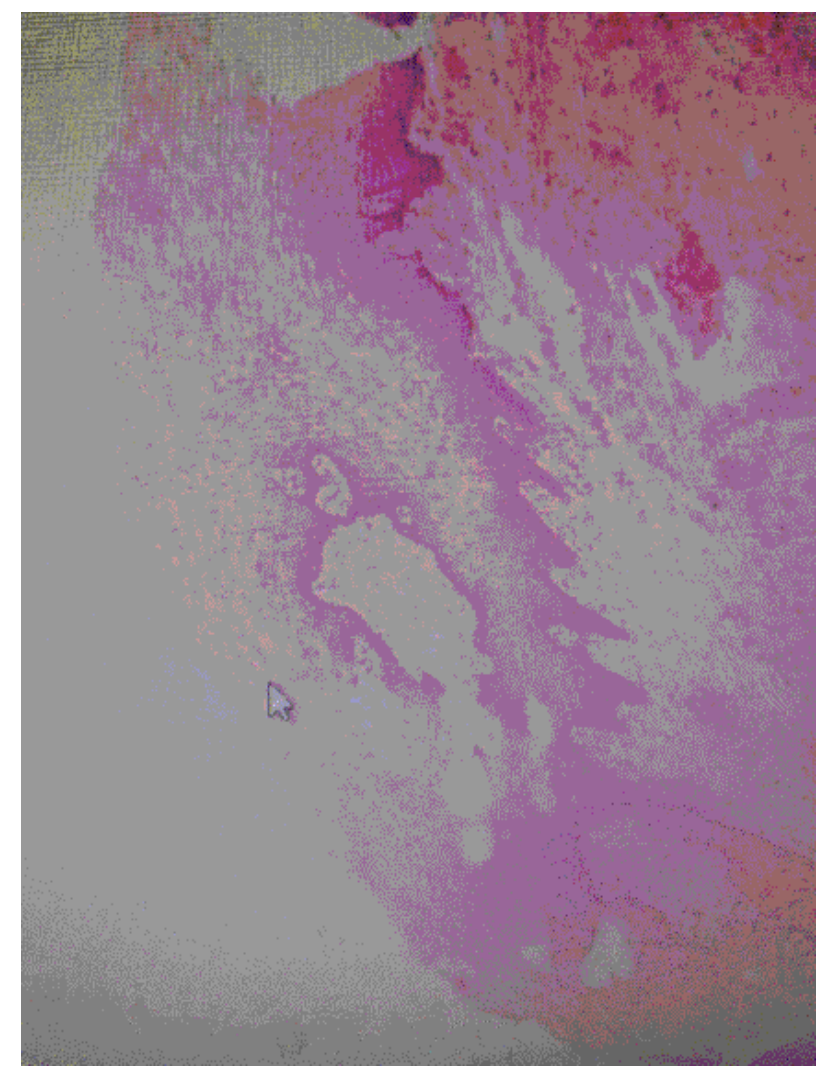

Figure 2: Vaginal sample at T4: Vaginal Histological modification induced by treatment. To be notice the thickening of the derma surface, the phenomenon of glycogenic acanthosis, the increased number and highness of papillae in chorion and the reduction in the inflammatory tissue infiltrate.
VHIS increased significantly after the completion of the study protocol, demonstrating that 4 is probably the optimal treatment's number. At the baseline (TO), 90\% of patients had a VHIS score $<15$ (atrophic epithelium). After three treatments, $74 \%$ of patients present a VHIS score $>15$. After the fourth last treatment, VHIS measures were further improved, demonstrating a restoration of vaginal epithelial tropism in $95 \%$ of treated women. These results have been already the aim of a previous paper focused specifically on symptoms [12]. Two of the patients who achieved inferior results on vaginal atrophy were in advanced age (76 and 79-yearsold) and the other two had had an early menopause before 40 years. This is a fundamental observation: To be effective laser treatment has to be offer not too late in time, when tissue changes are too deep to be restored. Probably, the optimal time offer is one year after menopause diagnosis, with one or more catch up treatments in the years to come.

The number of patients that at the beginning of the study had an FSFI score $\leq 26.55$ (corresponding to sexual dysfunction) dropped from $92 \%$ to $44 \%$ after two laser treatment. At the study protocol completed only $10 \%$ of women showed an FSFI score $\leq 26.55$, demonstrating that SSVL is effective in $90 \%$ of the treated cases if an adequate number of treatment is guaranteed.

As expected from the histological changes, also for urinary incontinence's symptoms an improvement was obtained: $91 \%$ of symptomatic patients showed complete benefit after SSVL treatment with no symptoms left, $97 \%$ of these were previously affected by urge-incontinence and $85 \%$ by stress-incontinence, all this underlining that the tissue modification may be the causative factor.

\section{Discussion}

This study assesses the effect of SSVL therapy in postmenopausal women who had failed to respond to estrogen therapy or had refused it, focusing on histological changes pre and post treatment and analyzing the GSM and the lower urinary tract related symptoms. This efficacy was assessed in both principles of treatment, improvement of local pathophysiology and consequently alleviation of symptoms, and it was independent to the participants' baseline characteristics (i.e years since

Table 2: GSM symptoms evaluated using VAS at T2 and T4.

\begin{tabular}{|c|c|c|c|}
\hline VAS & Baseline (T0) & 4 week FUP (T2) & 8 week FUP (T4) \\
\hline 0 & 0 & 0 & 0 \\
\hline 1 & 0 & $15 / 80$ (19\%) & $56 / 80(70 \%)$ \\
\hline $2-3$ & $4 / 80(5 \%)$ & $5 / 80(6 \%)$ & $12 / 80$ (15\%) \\
\hline $4-5$ & $8 / 80(10 \%)$ & $12 / 80(15 \%)$ & $8 / 80(10 \%)$ \\
\hline $6-7$ & $11 / 80(14 \%)$ & 25/80 (31\%) & 0 \\
\hline $8-9$ & $37 / 80(46 \%)$ & $17 / 80(21 \%)$ & $4 / 80(5 \%)$ \\
\hline 10 & $20 / 80(25 \%)$ & $6 / 80(8 \%)$ & 0 \\
\hline
\end{tabular}


last period).

Improvement in histological features was demonstrated after SSVL comparing tissue samples collected before and after treatment and at the same time an amelioration was observed on VHIS, VVA symptoms and sexual female function. These results were progressive with treatment going by and reached the highness after the forth laser session and were time lasting, been unvaried 4 week after the end.

Research has already demonstrated the tissue remodeling properties of fractional lasers in aesthetic medicine with the effect of stimulating the production of new collagen and elastin, bolstering the extracellular matrix [13]. Improvement of vulvovaginal symptoms after fractional laser has been demonstrated in multiple case series, though this is the first study to our knowledge that analyze the histological and inflammatory changes induced by SSVL laser treatment and their significance $[14,15]$.

The main contribution of this study is the use of a new type of laser, the Solid State Vaginal Laser (SSVL). This SSVL is based on a non-ablative procedure like to the radiofrequency effect. Its handpiece is characterized by a larger spot that doesn't focus the beam on surface, but deeply penetrate vaginal tissues, thus reaching that layers in which collagen fibers are more represented with greater effectiveness. It also avoids damaging mucosal surface, in opposition to what it happens using ablative and fractional ablative systems [16].

In this study we demonstrated that, after four laser treatments, histological modifications are present [17] and deep enough to be interpreted as tissue remodeling in a rejuvenating sense. In particular we observed first a reduction of fibrosis and the increase of collagenic and angioblastic fibroblastic activity (angioblastic granulation tissue-like). Furthermore, the coating of the epithelium is progressive and thickening of the derma surface can be observed together with the phenomenon of glycogenic acanthosis causing the "glycogenization". In addition, the chorion gained in number and highness of papillae hand in hand with reduction of fibrosis. The other key point is the demonstration of inflammatory tissue reduction: Comparing samples collected before and after treatment a decreasing in the lymphocytic and, in general in all inflammatory infiltrate of derma and chorion, can be observed and this infiltrate is almost absent after the last laser session, meaning a restitution ad integrum not only from an histological but also from an inflammation point of view [18]. This is probably one of the main point acting in the GSM symptoms reduction.

Two of the patients who achieved inferior results on vaginal atrophy were in advanced age (76 and 79-yearsold) and the other two had had an early menopause before 40 years. This suggest that laser treatment to be highly effective has to be offer not too late in time, when tissue changes are unlikely to be restored [19]. Probably, the optimal time offer is one year after menopause diagnosis, with one or more single catch up treatments in the years to come to balance the progressive mucosal ageing.

The SSVL equipment used in this study is based on a specific protocol, called Ladylift ${ }^{\circledR}$, performed with a laser with a wavelength of $1470 \mathrm{~nm}$ (Eufoton -TriesteItaly) transmitted with a specific probe and parameters customized according to the patient.

The common mechanism of UI is pelvic floor dysfunction due to loss of its supportive function. The mechanical stability of the urethra and bladder neck is largely provided by intact pelvic muscles and connective tissue of the pelvis. The mechanism of action of laser treatment for SUI is largely due to thickening and strengthening of the vaginal wall with an emphasis to the anterior wall, which supports the bladder and urethra. Our results suggest that VSSL working with sub necrotic temperatures, aim to influence collagen remodeling restoring the natural composition of a content pelvic floor [20].

However, this multicenter observational study has several limitations: Lack of long-term follow-up, lack of randomization with a sham treatment and/or standard treatment, and absence of a comparator (placebo or other active treatment).

Beside this, the histological results together with the improvement of GSM symptoms as indicated by VHIS, FSFI and ICIQ-UI SF scores prove a real favorable effect of SSVL on tissue remodeling and revitalization that ends in a 'rejuvenation' effect from both histological and symptomatic point of view.

\section{Acknowledgments}

None.

\section{Funding}

No funding

\section{Disclosure of Interest}

The authors declare no conflict of interest.

\section{References}

1. Portman DJ, Gass MLS (2014) Genitourinary syndrome of menopause: New terminology for vulvovaginal atrophy from the International Society for the Study of Women's Sexual Health and The North American Menopause Society. Menopause 21: 1063-1068.

2. Ward K, Deneris A (2016) Genitourinary syndrome of menopause: A new name for an old condition. Nurse Pract 41: 28-33.

3. Graziottin Alessandra, Murina Filippo (2017) Vulvar pain. From childhood to old age. Springer.

4. Havemann LM, Cool DR, Gagneux P, Markey MP, Yaklic JL, et al. (2017) Vulvodia: what we know and where we should be going. J Low Genit Tract Dis 2017: 150-156. 
5. Gandhi J, Chen A, Dagur G, Suh Y, Smith N, et al. (2016) Genitourinary syndrome of menopause: An overview of clinical manifestations, pathophysiology, etiology, evaluation, and management. Am J Obstet Gynecol 215: 704-711.

6. Johannesson $U$, Blomgren $B$, Hilliges $M$, Rylander $E$, Bohm-Starke N (2007) The vulvar vestibular mucosamorphological effects of oral contraceptives and menstrual cycle. Br J Dermatol 157: 487-493.

7. Nappi RE, Biglia N, Cagnacci A, Di Carlo C, Luisi S, et al. (2016) Diagnosis and management of symptoms associated with vulvovaginal atrophy: Expert opinion on behalf of the Italian VVA study group. Gynecol Endocrinol 32: 602-606.

8. Murina F, Karram M, Salvatore S, Felice R (2016) Fractional $\mathrm{CO}_{2}$ laser treatment of the vestibule for patients with vestibulodynia and genitourinary syndrome of menopause: A pilot study. J Sex Med 13: 1915-1917.

9. Bachmann GA (1994) Vulvo-vaginal complaints. In: Lobo $\mathrm{R}$, Treatment of the Postmenopausal Woman. Raven Press, New York.

10. Rosen R, Brown C, Heiman J, Leiblum S, Meston C, et al. (2000) The Female Sexual Function Index (FSFI): A multidimensional self-report instrument for the assessment of female sexual function. J Sex Marital Ther 26: 191-208.

11. Choi EP, Lam CL, Chin WY (2015) The test-retest reliability of the Incontinence Questionnaire-Urinary Incontinence Short Form (ICIQ-UI SF) for assessing type of urinary incontinence in males and females. J Clin Nurs 24: 37423744 .

12. D Dodero, F Frascani, M Angelucci, G Bernabei, $E$ Merlo, et al. Solid state vaginal laser for the treatment of Genitourinary Syndrome of Menopause: A preliminary report, Scirp in press.

13. Ong MW, Bashir SJ (2012) Fractional laser resurfacing for acne scars: A review. Br J Dermatol 166: 1160-1169.

14. Arunkalaivanan A, Kaur H, Onuma O (2017) Laser therapy as a treatment modality for genitourinary syndrome of menopause: a critical appraisal of evidence. Int Urogynecol J 28: 681-685.

15. Zdenko Vizintin, Mario Rivera, Ivan Fistonić, Ferit Saraçoğlu, Paolo Guimares, et al. (2012) Novel minimally invasive VSP Er: YAG laser treatments in gynecology. J Laser Health Acad 1: 46-58.

16. Pitsouni E, Grigoriadis T, Falagas ME, Salvatore S, Athanasiou S (2017) Laser therapy for the genitourinary syndrome of menopause. A systematic review and metaanalysis. Maturitas 103: 78-88.

17. Zerbinati N, Serati M, Origoni M, Candiani M, lannitti T, et al. (2015) Microscopic and ultrastructural modifications of postmenopausal atrophic vaginal mucosa after fractional carbon dioxide laser treatment. Lasers Med Sci 30: 429436.

18. Regauer S, Eberz B, Beham-Schmid C (2015) Mast cell infiltrates in vulvodynia represent secondary and idiopathic mast cell hyperplasias. APMIS 2015 123: 452-456.

19. Zhou L, Lee JH, Wen Y, Constantinou C, Yoshinobu M, et al. (2012) Biomechanical properties and associated collagen composition in vaginal tissue of women with pelvic organ prolapse. J Urol 188: 875-880.

20. Fistonić N, Fistonić I, Guštek ŠF, Turina IS, Marton I, et al. (2016) Minimally invasive, non-ablative Er: YAG laser treatment of stress urinary incontinence in women-a pilot study. Lasers Med Sci 31: 635-643. 